$\mathrm{O}_{2}{ }^{--}$production, MAPK and $\mathrm{NFkB}$ activation, and monocyte adhesion (all $\mathrm{p}<0.05$; one-way AVOVA). These C242T effects were further confirmed using $\mathrm{p} 22^{\text {phox }}$ shRNA engineered HeLa cells and coronary microvascular endothelial cells isolated from Nox2 knockout mice. Clinical significance was investigated using saphenous vein segments from non-CHD subjects after phlebectomies. Informed consents were obtained from the patients and the project was approved by the local NHS and the university ethical committees according to the UK regulation. TT $(\mathrm{C} 242 \mathrm{~T})$ allele was common (prevalence of $20 \%$ ) and compared to CC, veins bearing TT allele had significantly lower levels of Nox2 expression and $\mathrm{O}_{2}{ }^{--}$generation in response to high glucose challenge (all p < 0.05).

In summary, our study for the first time provides mechanistic insight into the protective effect of the $\mathrm{p} 22^{\text {phox }} \mathrm{C} 242 \mathrm{~T}$ SNP against the inflammatory oxidative stress-related cardiovascular diseases. $\mathrm{p} 22^{\text {phox }} \mathrm{C} 242 \mathrm{~T}$ SNP causes $\mathrm{p} 22^{\text {phox }}$ structural changes that alter its interaction with the catalytic subunit Nox2 and inhibits endothelial oxidative response to TNFalpha or high glucose stimulation.

\section{TARGETING THE HIPPO SIGNALLING PATHWAY TO ENHANCE THE THERAPEUTIC POTENTIAL OF IPS- DERIVED CARDIOMYOCYTES}

Abigail Robertson*, Elizabeth J Cartwright, Delvac Oceandy. University of Manchester; *Presenting Author

\subsection{6/heartjnl-2016-309890.168}

Introduction Cell based therapy using stem cell derived cardiomyocytes, has emerged as a potential therapeutic approach for cardiac diseases such as myocardial infarction and heart failure. Adult skin fibroblasts can be reprogrammed into induced pluripotent stem cells (iPSC) which could be an ideal source of iPS-derived cardiomyocytes (iPS-CM). Challenges facing cell therapy include the high number of viable cells needed to survive in pathological conditions. The Hippo signalling pathway has been described as a key pathway involved in regulating cardiomyocyte proliferation and survival in both embryonic and adult hearts. The purpose of this study is to test whether modification of the Hippo pathway will enhance the efficiency of iPS-CM generation and will increase iPS-CM survival and viability in pathological conditions.

Methods Skin fibroblasts were reprogrammed to iPS cells and then differentiated to cardiomyocytes. The Hippo signalling pathway was modified by genetic ablation of MST1, a major upstream regulator of the Hippo pathway, or by overexpressing YAP, the main downstream effector of the pathway. Cell proliferation was analysed using EdU incorporation assay and staining for cytokinesis markers Ki67 and phospho-histone H3. Cell death and viability were analysed using caspase $3 / 7$ and MTT activity and trypan blue staining in both normal and hypoxic conditions.

Results Analysis of cell proliferation shows that genetic ablation of Mst1 leads to significantly increased proliferation (12 $\pm 1.5 \% \mathrm{P}<0.001)$, survival and viability $(20 \pm 4.3 \% \mathrm{P}<$ 0.001 ) of iPSC in both normal and hypoxic conditions compared to controls. In addition overexpression of YAP, which is normally inhibited by upstream Hippo pathway components, and overexpression of mutated constitutively active form of YAP (S127A) increases cell proliferation in iPS-CM compared to control iPS-CM as shown with EdU assay $(+20.8 \pm 1.6 \%$
$\mathrm{P}<0.01)$ and Ki67 staining $(4.9 \pm 0.9 \% \mathrm{P}<0.001)$. Overexpression of YAP leads to up regulation of genes associated with inhibition of apoptosis and promotion of cell proliferation.

Conclusion Targeting the Hippo pathway in iPS cells and iPS$\mathrm{CM}$ significantly increases proliferation and survival in both normal and hypoxic conditions. Therefore, modulation of the Hippo pathway could become a new strategy to enhance the therapeutic potential of iPS-CM.

\section{SOLUBLE GUANYLATE CYCLASE ACTIVATORS AS COMBINATION ANTI-PLATELET THERAPY WITH P2Y12 INHIBITORS AND PDE INHIBITORS: IN VIVO AND EX VIVO STUDIES}

${ }^{1}$ Plinio Ferreira*, ${ }^{2}$ Paul Armstrong, ${ }^{2}$ Melissa Chan, ${ }^{2}$ Timothy Warner. ${ }^{1}$ University of SÃfO Paulo/William Harvey Research Institute; ${ }^{2}$ William Harvey Research Institute; *Presenting Author

10.1136/heartjnl-2016-309890.169

Methods Mice were pre-treated with vehicle, prasugrel (0.3 $\mathrm{mg} / \mathrm{kg})$, cinaciguat $(0.3 \mathrm{mg} / \mathrm{kg})+$ dipyridamole $(2.0 \mathrm{mg} / \mathrm{kg})$, or prasugrel + cinaciguat + dipyridamole (triple) and anesthetized. To measure in vivo thrombus formation, the carotid artery was then exposed and thrombosis induced by placement of a piece of filter paper saturated with $10 \%$ ferric chloride in contact with the adventitial surface of vessel for 3 minutes. Carotid artery blood flow was monitored by a Doppler probe with the time to form an occlusive thrombus being taken as the time required for blood to stop flowing completely for $>1$ minutes. Time to occlusion from different treatment groups was compared. To measure ex vivo platelet function, blood was taken from the vena cava of treated mice and aggregation of platelets in whole blood in response to arachidonic acid (AA) $1 \mathrm{mM}$, PAR-4 amide $30 \mu \mathrm{M}$, and collagen 10 $\mu \mathrm{g} / \mathrm{ml}$ was then determined using flow cytometry.

Results In mice treated with vehicle, prasugrel and cinaciguat + dipyridamole complete vessel occlusion occurred within 8 minutes. Conversely, triple combination of prasugrel + cinaciguat + dipyridamole blocked thrombus formation (time to occlusion $>24$ minutes). In ex vivo platelet function tests, we observed reduced platelet aggregation in mice treated with the triple combination compared to other treatments. Results as mean \pm SEM. AA; vehicle $83 \pm 9 \%$, prasugrel $67 \pm 7 \%$, cinaciguat + dipyridamole $62 \pm 9 \%$, triple $27 \pm 27 \%$ : PAR-

Prasugrel $(0.3 \mathrm{mg} / \mathrm{kg}) \&$ Dipyridamole $(2 \mathrm{mg} / \mathrm{kg}) \&$ Cinaciguat $(0.3 \mathrm{mg} / \mathrm{kg})$

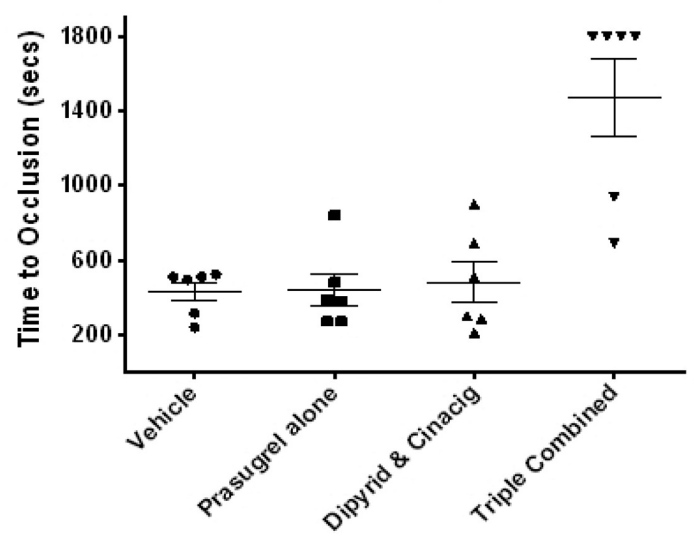

Abstract 169 Figure 1 